\title{
DETERMINAÇÃO DE PARACETAMOL EM PRODUTOS FARMACÊUTICOS USANDO UM BIOSSENSOR DE PASTA DE CARBONO MODIFICADO COM EXTRATO BRUTO DE ABOBRINHA (Cucurbita pepo)
}

\author{
Iolanda Cruz Vieira
}

Faculdade de Farmácia e Bioquímica, Universidade de Cuiabá, Av. Beira Rio, 3100, 78015-480 Cuiabá - MT

Karina Omuro Lupetti e Orlando Fatibello-Filho*

Departamento de Química, Centro de Ciências Exatas e de Tecnologia, Universidade Federal de São Carlos, CP 676, 13560-970

São Carlos - SP

Recebido em 29/1/02; aceito em 28/6/02

\begin{abstract}
DETERMINATION OF PARACETAMOL IN PHARMACEUTICAL PRODUCTS USING A CARBON PASTE BIOSENSOR MODIFIED WITH CRUDE EXTRACT OF ZUCCHINI (Cucurbita pepo). Crude extracts of several vegetables such as peach (Prunus persica), yam (Alocasia macrorhiza), manioc (Manihot utilissima), artichoke (Cynara scolymus L), sweet potato (Ipomoea batatas (L.) Lam.), turnip (Brassica campestre ssp. rapifera), horseradish (Armoracia rusticana) and zucchini (Cucurbita pepo) were investigated as the source of peroxidase (POD: EC 1.11.1.7). Among those, zucchini (Cucurbita pepo) crude extract was found to be the best one. This enzyme in the presence of hydrogen peroxide catalyses the oxidation of paracetamol to $\mathrm{N}$-acetylp-benzoquinoneimine which the electrochemical reduction back to paracetamol was obtained at a peak potential of $-0.10 \mathrm{~V}$. A cyclic voltammetric study was performed by scanning the potential from +0.5 to $-0.5 \mathrm{~V}$. The recovery of paracetamol from two samples ranged from 97.3 to $106 \%$ and a rectilinear calibration curve for paracetamol concentration from $1.2 \times 10^{-4}$ to $2.5 \times 10^{-3}$ mol L-1 $(r=0.9965)$ were obtained. The detection limit was $6.9 \times 10^{-5} \mathrm{~mol} \mathrm{~L}^{-1}$ and the relative standard deviation was less than $1.1 \%$ for a solution containing $2.5 \times 10^{-3} \mathrm{~mol} \mathrm{~L}^{-1}$ paracetamol and $2.0 \times 10^{-3} \mathrm{~mol} \mathrm{~L}^{-1}$ hydrogen peroxide $(\mathrm{n}=12)$. The results obtained for paracetamol in pharmaceutical products using the proposed biosensor and Pharmacopoeial procedures are in agreement at the $95 \%$ confidence level.
\end{abstract}

Keywords: crude extract; carbon paste; paracetamol.

\section{INTRODUÇÃo}

O paracetamol ou acetaminofenol (N-acetil-p-aminofenol) é amplamente utilizado como analgésico e antipirético, possuindo ação semelhante à aspirina. Foi inicialmente utilizado na medicina em 1893 por von Mering. Contudo, só se tornou popular a partir de 1949 quando foi reconhecido como o principal metabólito ativo tanto da acetanilida como da fenacetina, ambos com ação analgésicaantipirética ${ }^{1}$.

Apesar de suas propriedades farmacológicas, uma "overdose" de paracetamol pode causar sérias lesões hepáticas (10 a $15 \mathrm{~g})$ e até ser fatal (20 a $25 \mathrm{~g})^{1}$. Devido a esta possibilidade, determinações desse composto farmacêutico, tanto em fluidos biológicos como em medicamentos, têm sido realizadas através de diferentes procedimentos.

Os métodos de cromatografia em camada delgada (CCD) e cromatografia gás-líquido (CGL) são recomendados pela Farmacopéia Americana ${ }^{2}$. Métodos espectrofotométricos ${ }^{3}$ também são citados na literatura, além de métodos espectroeletroquímicos ${ }^{4}$ que são utilizados para determinação dos compostos, bem como acompanhamento dos mecanismos de reação, além dos procedimentos de análise por injeção em fluxo ${ }^{5}$ e voltamétricos ${ }^{6,7}$.

O primeiro eletrodo de pasta de carbono ${ }^{8}$ foi desenvolvido, em 1958, na tentativa de preparar um eletrodo de carbono renovável para ser usado em uma ampla faixa de potenciais positivos onde o eletrodo de mercúrio metálico não é aplicável devido à sua oxidação. No entanto, o primeiro eletrodo de pasta de carbono modificado com enzima (glicose oxidase) foi proposto apenas no final da déca-

*e-mail: bello@dq.ufscar.br da de $80^{9}$, e desde então vários biossensores foram desenvolvidos usando diversos materiais biológicos ${ }^{10-16}$.

A pasta de carbono é uma mistura de pó de grafite e aglutinante. O pó de grafite deve ter tamanho uniforme, alta pureza química e baixa capacidade de adsorção de oxigênio e impurezas eletroativas. $\mathrm{O}$ aglutinante é geralmente um líquido orgânico quimicamente inerte, de baixa volatilidade, livre de impurezas, eletroinativo, imiscível com a solução do analito e deve preencher os interstícios entre as partículas do grafite. O desenvolvimento do eletrodo de pasta de carbono modificado com enzimas (biossensores) possui a vantagem de diminuir o limite de detecção do analito de interesse e aumentar a seletividade das determinações. O procedimento é simples e a regeneração da superfície é feita através da renovação da pasta da ponta do biossensor. Excelentes revisões sobre eletrodos de pasta de carbono e eletrodos modificados com peroxidase foram recentemente publicadas $^{14-17}$.

A peroxidase na presença de peróxido de hidrogênio catalisa a oxidação de alguns substratos doadores de prótons como mono e difenóis, polifenóis, aminofenóis entre outros. Essa enzima é termoestável e pode ter sua atividade regenerada após tratamento térmico. Diversas são as fontes vegetais de peroxidase como pêssego (Prunus persica), inhame (Alocasia macrorhiza), mandioca (Manihot utilissima), alcachofra (Cynara scolymus L.), batata doce (Ipomoea batatas (L.) Lam.), nabo (Brassica campestre ssp. rapifera), rabanete (Armoracia rusticana), abobrinha (Cucurbita pepo) e outras ${ }^{10,18-20}$.

O Brasil possui uma grande variedade de vegetais que podem constituir em uma fonte inesgotável de enzimas para serem aplicadas nas mais diversas áreas do conhecimento. Em química analítica por exemplo, elas podem ser usadas na construção de biossensores e/ou outros procedimentos enzimáticos ${ }^{10,21-29}$. Ademais, há uma tendência mundial de se usar extratos brutos e/ou tecidos de vegetais no lugar de enzimas 
purificadas em procedimentos bioanalíticos. O uso de extratos brutos pode apresentar em alguns casos, certa desvantagem na seletividade do método analítico, mas por outro lado é extremamente econômico, simples e geralmente possuem um tempo de vida superior a aqueles métodos que utilizam enzimas purificadas, visto que estas enzimas naturalmente imobilizadas nas células destes materiais biológicos (seu habitat natural) são mais estáveis. Além dessas vantagens, esses procedimentos geralmente não necessitam de cofatores, pois os extratos brutos empregados normalmente já possuem cofatores naturais para a reação enzimática do analito de interesse.

No presente trabalho, estudou-se inicialmente as condições de obtenção do extrato bruto de interesse como $\mathrm{pH}$, agentes protetores e/ou estabilizantes, além do tempo de armazenamento de diversos extratos enzimáticos. Após otimização do processo de extração, foram selecionados diferentes tecidos vegetais como pêssego, inhame, mandioca, alcachofra, batata doce, nabo, rabanete e abobrinha e determinadas as atividades dos extratos brutos enzimáticos. Em seguida, construiu-se um eletrodo de pasta de carbono modificado com extrato bruto de abobrinha, fonte da enzima peroxidase (POD: EC 1.11.1.7) e determinou-se o paracetamol em produtos farmacêuticos.

\section{PARTE EXPERIMENTAL}

\section{Equipamentos}

Os tecidos vegetais usados na obtenção do extrato bruto contendo a enzima peroxidase foram homogeneizados em um liquidificador da Walita, modelo Firenze RI6755. Usou-se na centrifugação do material biológico vegetal, uma centrifuga Du Pont Instrumentos Sorvall modelo RC-5B, provida de rotor modelo SS-34 com diâmetro de $23 \mathrm{~cm}$.

As medidas espectrofotométricas para determinação de proteína total e atividade dos extratos brutos foram feitas empregando-se um espectrofotômetro Femto modelo 434, utilizando-se cubetas de quartzo de $1,00 \mathrm{~cm}$ de caminho ótico.

O eletrodo de trabalho utilizado foi o de pasta de carbono modificado com extrato bruto de abobrinha (Cucurbita pepo), embutida em uma seringa para insulina com capacidade de $1 \mathrm{~mL}$. O contraeletrodo usado foi de platina com área de $1 \mathrm{~cm}$ x $0,5 \mathrm{~cm}$ e o de referência de prata-cloreto de prata $(\mathrm{Ag} / \mathrm{AgCl})$ e solução interna de $\mathrm{NaCl} 0,1 \mathrm{~mol} \mathrm{~L}^{-1}$ e $\mathrm{NaNO}_{3} 1,0 \mathrm{~mol} \mathrm{~L}^{-1}$, separada da solução de trabalho por uma placa porosa ultra fina de Vycor.

As medidas ciclovoltamétricas foram feitas em uma célula de vidro sem compartimento divisório, de capacidade de $20 \mathrm{~mL}$, com tampa de PVC com orifícios circulares para encaixe dos eletrodos e adição das soluções, utilizando-se um Potenciostato/Galvanostato, PAR modelo 173 EG\&G.

\section{Reagentes e soluções}

Reagentes de grau analítico foram usados para preparar as soluções tampão e de referência. Guaiacol, peróxido de hidrogênio, albumina de soro bovino e paracetamol foram adquiridas da Sigma Chemical Co (St. Louis, Mo, USA). Todas as soluções foram preparadas diariamente em tampão fosfato $0,1 \mathrm{~mol} \mathrm{~L}^{-1}$ (pH 6,0). Para construção do eletrodo de pasta de carbono foi usado pó de grafite (Acheson-38) da Fisher e óleo mineral (Nujol) da Aldrich.

Foram determinados o teor de paracetamol nas amostras de Resfenol, Resprin, Tylenol e Vick Pyrena, obtidas no comércio da cidade de São Carlos-SP.

\section{Obtenção do extrato bruto enzimático}

As abobrinhas utilizadas em todo este trabalho foram adquiridas em uma chácara da região de São Carlos. Com a finalidade de usar o mesmo produto, selecionou-se uma área do plantio exclusivamente para retirada desse material biológico. Após lavagem e secagem, $25 \mathrm{~g}$ de abobrinhas descascadas foram picadas e homogeneizadas em um liquidificador com $100 \mathrm{~mL}$ de tampão fosfato $0,1 \mathrm{~mol} \mathrm{~L}^{-1}$ (pH 6,5), contendo 2,5 g de agente protetor (Polyclar Super R) ${ }^{23-27}$. Em seguida, o homogenato foi filtrado em quatro camadas de tecido (gaze) e centrifugado a 25000xg (18000 rpm) durante $20 \mathrm{~min}$, a $4{ }^{\circ} \mathrm{C}$. A solução sobrenadante foi dividida em diversas alíquotas, armazenadas em refrigerador a $4{ }^{\circ} \mathrm{C}$ e usadas como fonte enzimática da peroxidase ${ }^{28}$. Os demais extratos brutos foram obtidos usando-se o mesmo procedimento.

\section{Determinação da atividade e proteína total da enzima peroxidase}

A atividade da peroxidase foi determinada medindo-se a variação de absorbância $(\lambda=470 \mathrm{~nm})$ do tetraguaiacol formado na reação enzimática. Nessa determinação foram usados $0,2 \mathrm{~mL}$ da solução sobrenadante (homogenato), $2,7 \mathrm{~mL}$ da solução de guaiacol $0,05 \mathrm{~mol} \mathrm{~L}^{-1}$ e $0,1 \mathrm{~mL}$ da solução de peróxido de hidrogênio $10,3 \mathrm{mmol} \mathrm{L}^{-1}$ em tampão fosfato $0,1 \mathrm{~mol} \mathrm{~L}^{-1}(\mathrm{pH} 6,5)$, a $25^{\circ} \mathrm{C}^{28}$. Uma unidade de atividade (unidades $\mathrm{mL}^{-1}$ ) é definida como a quantidade de enzima que causa o aumento de 0,001 unidades de absorbância por min nas condições mencionadas ${ }^{23-29}$.

O teor de proteína total da solução sobrenadante foi determinado pelo método do biureto $^{30}$, empregando-se albumina de soro bovino como padrão.

\section{Construção do eletrodo de pasta de carbono}

Dois tipos de eletrodos de pasta de carbono foram construídos: o primeiro, sem modificador e o segundo modificado com extrato bruto de abobrinha. O eletrodo de pasta de carbono sem enzima foi obtido pela homogeneização de diferentes composições em massa de pó de grafite $(65,0 ; 70,0 ; 72,5 ; 74,0$ e 75,0\%), Nujol $(34,0 ; 29,0$; 26,$5 ; 25,0$ e $24,0 \%$ ) e $1 \%$ de albumina de soro bovino em almofariz com pistilo durante $20 \mathrm{~min}$. Em seguida, esta pasta foi armazenada em um dessecador a $4{ }^{\circ} \mathrm{C}$ durante $15 \mathrm{~h}$.

A pasta modificada com extrato bruto de abobrinha foi obtida homogeneizando-se $0,375 \mathrm{~g}$ de pó de grafite e diferentes concentrações da enzima $(20 ; 50 ; 80 ; 100 ; 200 ; 300$; e 500 unidades peroxidase mg de proteína ${ }^{-1}$ ) durante $20 \mathrm{~min}$. Em seguida, foi adicionado nesta mistura $0,120 \mathrm{~g}$ de Nujol, sendo então a pasta homogeneizada por mais 20 min. Essa pasta foi armazenada em um dessecador na mesma temperatura e tempo supramencionados. Finalmente, as pastas foram embutidas em seringas e usadas como eletrodos de trabalho.

\section{Preparação das amostras}

Mediu-se acuradamente uma massa de cada fármaco no intervalo de 5-6 g, transferiu-se para um balão volumétrico de $50 \mathrm{~mL}$ e dissolveu-se em tampão fosfato $0,1 \mathrm{~mol} \mathrm{~L}^{-1}, \mathrm{pH}$ 6,5. Em seguida, uma alíquota de $1 \mathrm{~mL}$ foi transferida para uma célula contendo $10 \mathrm{~mL}$ de tampão fosfato $0,1 \mathrm{~mol} \mathrm{~L}^{-1}(\mathrm{pH} 6,5)$ com peróxido de hidrogênio $2,0 \times 10^{-3} \mathrm{~mol} \mathrm{~L}^{-1}$ e determinado o teor de paracetamol pelo método de adição múltipla de padrão.

\section{RESULTADOS E DISCUSSÃO}

\section{Obtenção do extrato bruto enzimático}

A atividade e a proteína total do extrato bruto enzimático variam 
em função do procedimento de extração escolhido e também do meio utilizado. Desta maneira, realizou-se um estudo sistemático de extração da peroxidase de diversos tecidos vegetais. Neste estudo, investigou-se o efeito do $\mathrm{pH}$, de vários agentes protetores e tempo de armazenamento. Após otimização das condições de extração, foram selecionados os tecidos vegetais de pêssego (Prunus persica), inhame (Alocasia macrorhiza), mandioca (Manihot utilissima), alcachofra (Cynara scolymus L.), batata doce (Ipomoea batatas (L.) Lam.), nabo (Brassica campestre ssp. rapifera), rabanete (Armoracia rusticana) e abobrinha (Cucurbita pepo) e determinou-se a atividade específica de cada extrato bruto enzimático.

Inicialmente, estudou-se o efeito do $\mathrm{pH}$ variando de 5,0 a 8,0 sobre a extração da peroxidase daqueles tecidos vegetais. Neste estudo, fixou-se uma quantidade de 2,5 g de agente protetor Polyclar SB-100, 25,0 g de tecido vegetal e $100 \mathrm{~mL}$ de tampão, variando-se o pH da solução tampão no intervalo de 5-8, sendo a atividade dessas soluções determinadas nos valores de $\mathrm{pH}$ de 5,0 a 8,0. A maior atividade enzimática foi obtida em $\mathrm{pH}$ de extração 6,5 e pH de determinação da atividade 6,0 , sendo assim selecionados.

Sabe-se da literatura ${ }^{22-29,31-34}$ que a tendência de escurecimento dos tecidos vegetais está relacionada com as reações entre a enzima polifenol oxidase ou peroxidase e os substratos naturais presentes nesses tecidos, na presença de oxigênio molecular. Com o objetivo de minimizar esses efeitos, diversas substâncias protetoras e/ou estabilizadoras como Polyclar Super R, Polyclar RJ, Polyclar K-30, Polyclar 10, Polyclar AT, Polyclar R e Polyclar SB-100 foram investigadas. Dessas substâncias, as maiores atividades específicas foram obtidas usando-se Polyclar Super R na razão de concentração de 2,5:25,0 m/m (Polyclar:tecido vegetal). Resultados similares foram obtidos em estudos anteriores desenvolvidos em nosso grupo de pesquisa $^{23-27}$.

Polyclar Super R é o polímero polivilpirrolidona (PVP) de alta massa molar, comercializado na forma de pó branco de baixa granulometria. Esse polímero é capaz de remover compostos fenólicos de soluções, devido à sua baixa solubilidade e tem sido usado com sucesso pelo nosso grupo de pesquisa ${ }^{23-28}$ para separar compostos fenólicos naturais de diversos extratos enzimáticos. Este seu bom desempenho é atribuído à formação de pontes de hidrogênio ${ }^{31}$ entre os substratos e o polímero PVP. Outras substâncias e polímeros também têm sido usados para remoção dos compostos fenólicos durante a obtenção dos extratos brutos como resinas de troca iônica, Lcisteína, policaprolactam e polietileno glicol $^{22-29,31-36}$, entretanto estudos preliminares ${ }^{23-27}$ mostraram o melhor desempenho daquele polímero usado no presente trabalho.

Os extratos brutos obtidos da abobrinha, rabanete, batata doce e nabo, em Polyclar Super R foram estocados em um refrigerador a $4{ }^{\circ} \mathrm{C}$ e suas atividades foram determinadas periodicamente durante vários dias. O estudo do tempo de armazenamento desses extratos é mostrado na Figura 1. Como pode ser observado, o emprego de
Polyclar Super R mantém a atividade da peroxidase nesses vegetais durante vários meses, mostrando boa eficiência na remoção dos substratos naturais dos extratos brutos, além do baixo custo deste processo.

\section{Atividade enzimática de diferentes tecidos vegetais}

A Tabela 1 apresenta os valores encontrados de atividade, proteína total e atividade específica da enzima peroxidase dos diferentes tecidos vegetais selecionados anteriormente. Nesse estudo foram utilizadas uma solução de guaiacol $0,05 \mathrm{~mol} \mathrm{~L}^{-1} \mathrm{em}$ tampão fosfato $0,1 \mathrm{~mol} \mathrm{~L}^{-1}$ (pH 6,0) e uma solução de peróxido de hidrogênio 10,3 $\mathrm{mmol} \mathrm{L}{ }^{-1}$. As maiores atividades específicas foram encontradas para nabo, rabanete e abobrinha. Levando-se em consideração os resultados obtidos nesse estudo e aqueles de estabilidade (tempo de armazenamento), escolheu-se o extrato bruto da abobrinha para o desenvolvimento do biossensor de pasta de carbono.

\section{Eletrodos de pasta de carbono e pasta de carbono modificado com extrato bruto de abobrinha}

Inicialmente, foram estudadas diferentes composições de grafite e Nujol $(\% \mathrm{~m} / \mathrm{m})$ sobre a resposta do eletrodo de pasta de carbono. Foram investigadas 5 composições: 65,$0 ; 70,0 ; 72,5 ; 74,0$ e 75,0\% de pó de grafite e 34,0;29,0;26,5; 25,0 e 24,0\% de Nujol e $1 \%$ de albumina de soro bovino. O melhor desempenho analítico do eletrodo de pasta de carbono foi observado para aquele eletrodo contendo $75 \% \mathrm{~m} / \mathrm{m}$ de grafite, $24 \% \mathrm{~m} / \mathrm{m}$ de Nujol e $1 \%$ de albumina de soro

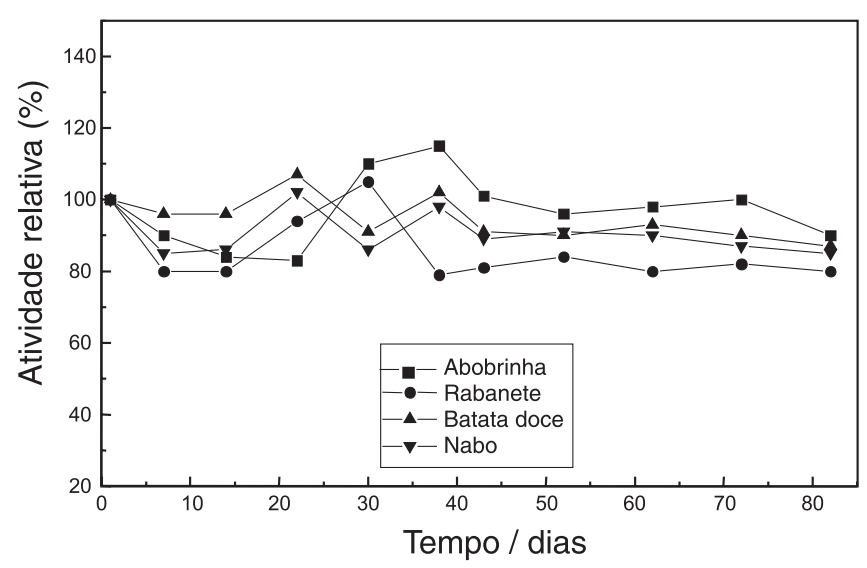

Figura 1. Estudo do tempo de armazenamento $\left(4^{\circ} \mathrm{C}\right)$ dos extratos brutos de abobrinha $(\mathbf{\square})$, rabanete $(\mathbf{O})$, batata doce $(\mathbf{\Delta})$ e nabo $(\boldsymbol{\nabla})$ em tampão fosfato $0,1 \mathrm{~mol} \mathrm{~L}^{-1}(\mathrm{pH} \mathrm{6,0)}$

Tabela 1. Atividade, proteína total e atividade específica da peroxidase encontrada em extrato bruto de diferentes tecidos vegetais

\begin{tabular}{lccr}
\hline Tecido vegetal & $\begin{array}{c}\text { Atividade } \\
\left.\text { (unidades } \mathrm{mL}^{-1}\right)\end{array}$ & $\begin{array}{c}\text { Proteína total } \\
\left(\mathrm{mg} \mathrm{mL}^{-1}\right)\end{array}$ & $\begin{array}{c}\text { Atividade específica } \\
\text { (unidades/mg de proteína)x10 }\end{array}$ \\
\hline Pêssego & 262 & 2,45 & 1,07 \\
Inhame & 1929 & 5,84 & 3,30 \\
Mandioca & 1738 & 2,67 & 6,51 \\
Alcachofra & 8905 & 4,61 & 19,3 \\
Batata Doce & 8688 & 3,21 & 27,1 \\
Nabo & 4226 & 0,490 & 86,2 \\
Rabanete & 3381 & 0,330 & 102 \\
Abobrinha & 6726 & 0,490 & 137 \\
\hline
\end{tabular}


bovino. Portanto, a pasta de carbono contendo essa composição de pó de grafite e Nujol foi empregada na construção dos biossensores contendo extrato bruto de abobrinha.

A velocidade de uma reação catalisada por enzima depende diretamente de sua concentração, portanto, após definição da melhor composição de grafite e Nujol $(75: 24 \% \mathrm{~m} / \mathrm{m})$, estudou-se a modificação da pasta de carbono, adicionando-se diferentes concentrações de enzima $(20 ; 50 ; 80 ; 100 ; 200 ; 300$ e 500 unidades peroxidase $\mathrm{mg}$ de proteína $\left.{ }^{-1}\right)(1 \% \mathrm{~m} / \mathrm{m})$. A melhor resposta desse biossensor foi obtida usando 200 unidades de peroxidase (400 unidades/g de pasta de carbono), decrescendo em concentrações superiores. Nas concentrações superiores dessa enzima, há um aumento da resistência elétrica da pasta de carbono contida em cada eletrodo, dificultando assim o processo de transferência de elétrons no biossensor, levando a um decréscimo da corrente de pico catódico.

\section{Efeito do pH e da concentração do peróxido de hidrogênio}

$\mathrm{O}$ pH exerce grande influência sobre as reações enzimáticas. As enzimas apresentam $\mathrm{pH}$ ótimo onde a atividade e a velocidade das reações são máximas. Sendo assim, estudou-se o efeito do $\mathrm{pH}$ de 5,0 a 7,5 sobre a resposta do biossensor de pasta de carbono. A melhor resposta do biossensor foi verificada em $\mathrm{pH}$ 6,0, decrescendo em valores de $\mathrm{pH}$ inferiores e superiores. Conseqüentemente, os estudos foram realizados em tampão fosfato $0,1 \mathrm{~mol} \mathrm{~L}^{-1}(\mathrm{pH} 6,0)$.

A enzima peroxidase catalisa a reação de oxidação de substratos doadores de prótons na presença de solução de peróxido de hidrogê-

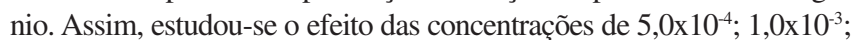
$2,0 \times 10^{-3} ; 4,0 \times 10^{-3} ; 6,2 \times 10^{-3} ; 8,2 \times 10^{-3}$ e $1,2 \times 10^{-2} \mathrm{~mol} \mathrm{~L}^{-1}$ de peróxido de hidrogênio sobre a oxidação de $4,0 \times 10^{-3} \mathrm{~mol} \mathrm{~L}^{-1}$ de paracetamol utilizando-se o biossensor descrito anteriormente $(75: 24: 1 \% \mathrm{~m} / \mathrm{m}$ grafite: Nujol: extrato bruto enzimático). A resposta analítica do biossensor cresceu até a concentração de $2,0 \times 10^{-3} \mathrm{~mol} \mathrm{~L}^{-1}$, mantendo-se então constante até a concentração de $1,2 \times 10^{-2} \mathrm{~mol} \mathrm{~L}^{-1}$. Selecionou-se então a concentração de $2,0 \times 10^{-3} \mathrm{~mol} \mathrm{~L}^{-1}$ de peróxido de hidrogênio para o desenvolvimento deste trabalho.

\section{Estudo dos substratos fenólicos e repetibilidade}

Determinou-se a resposta relativa do biossensor de pasta de carbono modificado com extrato bruto de abobrinha para diferentes substratos doadores de prótons como paracetamol, hidroquinona, isoproterenol, aldeído protocatecóico na concentração de 4,0x $10^{-3} \mathrm{~mol} \mathrm{~L}^{-1}$ e solução de peróxido de hidrogênio $2,0 \times 10^{-3} \mathrm{~mol} \mathrm{~L}^{-1} \mathrm{em}$ tampão fosfato $0,1 \mathrm{~mol} \mathrm{~L}^{-1}(\mathrm{pH} 6,0)$. Neste estudo, o biossensor mostrou-se mais sensível para paracetamol (100\%), seguido de hidroquinona $(94,5 \%)$, dopamina $(79,4 \%)$, isoproterenol $(76,9 \%)$ e aldeído protocatecóico $(35,3 \%)$.

No estudo da repetibilidade foram feitas 12 determinações para

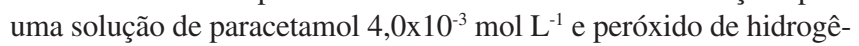
nio $2,0 \times 10^{-3} \mathrm{~mol} \mathrm{~L}^{-1}$ em tampão fosfato $0,1 \mathrm{~mol} \mathrm{~L}^{-1}(\mathrm{pH} 6,0)$. Uma corrente de pico catódica média de $82,8 \pm 0,9 \mu \mathrm{A}$ e um desvio padrão relativo de $1,1 \%$ foram obtidos, indicando assim boa repetibilidade do biossensor desenvolvido.

\section{Reação do paracetamol com peróxido de hidrogênio e peroxidase}

A peroxidase na presença de peróxido de hidrogênio catalisa a oxidação do paracetamol levando à formação de N-acetil-pbenzoquinonaimina. Em seguida, esse composto é reduzido eletroquimicamente ao substrato de partida, como representado na Figura 2.

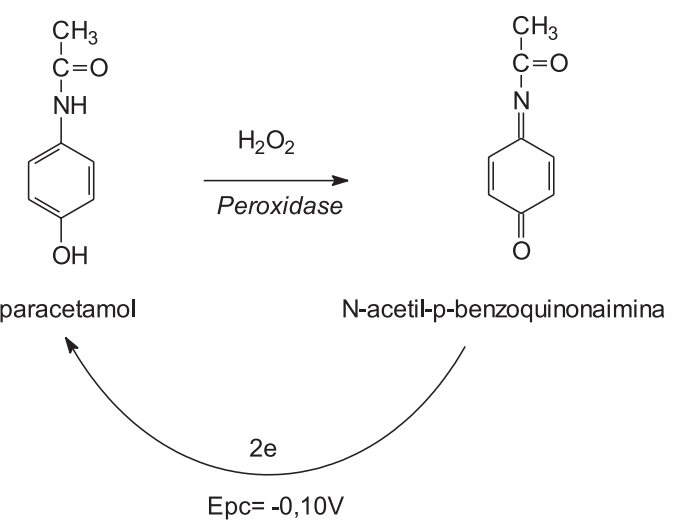

Figura 2. Representação esquemática das reações entre paracetamol, peróxido de hidrogênio e peroxidase no biossensor

Neste trabalho, as medidas ciclovoltamétricas usando o eletrodo de pasta de carbono foram feitas no potencial de $+0,5 \mathrm{a}-0,5 \mathrm{~V}$ com uma velocidade de varredura de $100 \mathrm{mV} / \mathrm{s}$, como apresentado na Figura 3. A curva analítica $\left(\mathrm{I}_{\mathrm{pc}}=3,115+15086\right.$ [paracetamol], $\left.\mathrm{r}=0,9965\right)$ foi construída a partir das correntes de pico catódicas (Ipc), medidas no potencial de $-0,10 \mathrm{~V}$, em função da concentração de paracetamol. Obteve-se uma relação linear entre as correntes de pico catódicas $(\mu \mathrm{A})$ e as concentrações de paracetamol no intervalo de $1,2 \times 10^{-4}$ a $2,5 \times$ $10^{-3} \mathrm{~mol} \mathrm{~L}^{-1}$ e um limite de detecção de $6,9 \times 10^{-5} \mathrm{~mol} \mathrm{~L}^{-1}$.

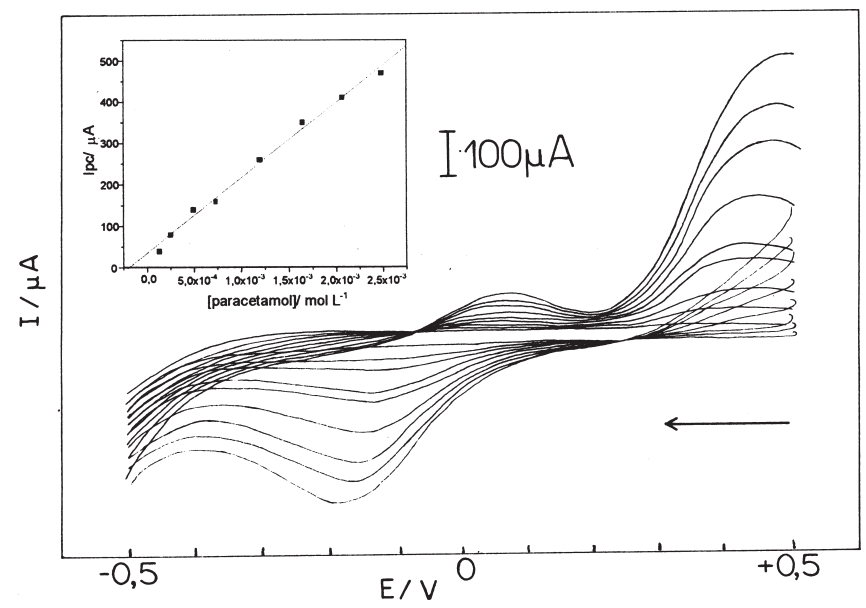

Figura 3. Voltamogramas cíclicos obtidos para soluções de paracetamol nas concentrações de $1,2 \times 10^{-4} ; 2,5 \times 10^{-4} ; 4,9 \times 10^{-4} ; 7,3 \times 10^{-4} ; 1,2 \times 10^{-3} ; 1,6$ $x 10^{-3} ; 2,1 \times 10^{-3}$ e 2,5 $\times 10^{-3} \mathrm{~mol} \mathrm{~L}^{-1}$ e peróxido de hidrogênio $2,0 \times 10^{-3} \mathrm{~mol} \mathrm{~L}^{-1}$ em tampão fosfato $0,1 \mathrm{~mol} \mathrm{L^{-1 }}$ (pH 6,0), a $25{ }^{\circ} \mathrm{C}$ e curva analítica para o paracetamol obtida com o biossensor proposto

\section{Efeito de substâncias concomitantes}

O efeito de substâncias concomitantes ou excipientes, freqüientemente encontradas nas amostras de paracetamol como ácido cítrico, benzoato de sódio, metabissulfito de sódio, estearato de magnésio e sacarina, foi avaliado utilizando o biossensor proposto. As razões de concentração entre paracetamol e os excipientes foram fixadas em $0,1,1,0$ e 10 . Nenhuma dessas substâncias interferiu na resposta do biossensor com exceção do metabissulfito em proporções 10 vezes maiores. No entanto, essa relação de concentrações não é encontrada em nenhum dos produtos farmacêuticos empregados nesse trabalho. 


\section{Estudos de adição e recuperação}

Os resultados obtidos no estudo de adição e recuperação de paracetamol em amostras de Resfenol e Resprin ( $n=5)$ estão apresentados na Tabela 2. Para esse estudo foram adicionadas em cada amostra $0,80,1,50,2,30$ e $3,00 \mathrm{mg} \mathrm{L}^{-1}$ de solução de paracetamol

Tabela 2. Estudo de adição e recuperação de paracetamol em produtos farmacêuticos

\begin{tabular}{lccc}
\hline \multirow{2}{*}{ Amostra } & \multicolumn{2}{c}{ Paracetamol $\left(\mathrm{mg} \mathrm{L}^{-1}\right)$} & \\
\cline { 2 - 3 } & Adicionado & Recuperado & Recuperação (\%) \\
\hline \multirow{3}{*}{ Resfenol } & 0,80 & $0,80 \pm 0,01$ & 100 \\
& 1,50 & $1,48 \pm 0,02$ & 98,7 \\
& 2,30 & $2,45 \pm 0,01$ & 106 \\
\multirow{3}{*}{ Resprin } & 3,00 & $2,92 \pm 0,05$ & 97,3 \\
& 0,80 & $0,80 \pm 0,02$ & 100 \\
& 1,50 & $1,50 \pm 0,01$ & 100 \\
& 2,30 & $2,37 \pm 0,01$ & 103 \\
& 3,00 & $3,07 \pm 0,02$ & 102 \\
\hline
\end{tabular}

$\mathrm{n}=5$ padrão, seguido da determinação desse fármaco e o cálculo da percentagem de recuperação. Os valores de recuperação de paracetamol nestas amostras variaram de 97,3 a 106\% indicando, assim, que o procedimento analítico proposto não sofre interferência das matrizes dessas amostras.

\section{Determinação de paracetamol em produtos farmacêuticos}

Para avaliar o desempenho do biossensor, determinou-se a concentração de paracetamol $\left(\mathrm{mg} \mathrm{g}^{-1}\right)$ nos produtos farmacêuticos Resfenol, Resprin, Tylenol e Vick Pyrena. A Tabela 3 apresenta uma comparação entre os teores de paracetamol obtidos pelos procedimentos proposto, da Farmacopéia ${ }^{2}$ e os valores rotulados. Os teores de paracetamol encontrados estão em concordância com aqueles teores de paracetamol rotulados a um nível de $95 \%$ de confiança e dentro de um intervalo de erros aceitável. O biossensor apresentou um bom desempenho analítico e um tempo de vida superior a 7 meses (mais de 900 determinações por quantidade de pasta embutida em cada seringa). Cabe ressaltar que o emprego desse extrato bruto vegetal proporcionou um tempo de vida do biossensor muito superior a aqueles tempos de vida de biossensores de pasta de carbono usando peroxidase purificada ${ }^{14}$.

Tabela 3 - Determinação de paracetamol em produtos farmacêuticos

\begin{tabular}{|c|c|c|c|c|c|}
\hline \multirow[b]{2}{*}{ Amostra } & \multicolumn{3}{|c|}{ Paracetamol (mg g-1) } & \multicolumn{2}{|c|}{ Erro relativo $(\%)$} \\
\hline & Valor rotulado & Farmacopéia & Biossensor & $\mathrm{E}_{1}$ & $\mathrm{E}_{2}$ \\
\hline Resfenol & 100 & $100,1 \pm 0,2$ & $108,5 \pm 0,1$ & $+8,5$ & $+8,4$ \\
\hline Resprin & 200 & $201,1 \pm 0,2$ & $208,4 \pm 0,1$ & $+4,0$ & $+3,5$ \\
\hline Tylenol & 200 & $199,7 \pm 0,3$ & $204,1 \pm 0,2$ & $+2,0$ & $+2,2$ \\
\hline Vick Pyrena & 500 & $500,1 \pm 0,1$ & $505,5 \pm 0,1$ & $+1,1$ & $+1,1$ \\
\hline
\end{tabular}

$\mathrm{n}=6$, limite de confiança $95 \% ; \mathrm{E}_{1}=$ erro relativo entre biossensor e valor rotulado; $\mathrm{E}_{2}=$ erro relativo entre biossensor e da farmacopéia.

\section{CONCLUSÕES}

O biossensor de pasta de carbono modificado com extrato bruto de abobrinha para determinação de paracetamol em produtos farmacêuticos desenvolvido neste trabalho possui várias vantagens como baixo custo, facilidade de construção, alta estabilidade e tempo de vida superior aos biossensores usando enzima purificada. A metodologia proposta é simples e poderá ser uma alternativa aos métodos cromatográficos de determinação desse fármaco.

\section{AGRADECIMENTOS}

Os autores agradecem o suporte financeiro das agências PADCT/ CNPq, CNPq, UNIC e FAPESP pela bolsa de mestrado de K. O. Lupetti.

\section{REFERÊNCIAS}

1. Gilman, A. G.; Rall, T. W.; Nies, A. S.; Palmer, T.; As Bases Farmacológicas da Terapêutica, 8ae ed., Guanabara Koogan: Rio de Janeiro, 1991.

2. US Pharmacopeial Convention, INC; United States Pharmacopeia- The National Formulary XXXIII, Twinbrook Parkway: Rockville, 1995, p.16.

3. Datta, B. K.; Bachar, S. C.; Banoo, R.; Haque, S.; Indian J. Pharm. Sci. 1989, 51, 270.

4. Fang, Y. Z.; Long, D. J.; Ye, J. N.; Anal. Chim. Acta 1997, 342, 13.

5. Verma, K. K.; Jain, A.; Stewart, K.K.; Anal. Chim. Acta 1992, 261, 261.

6. Huang, S. S.; Tang, H.; Li, B. F.; Mikrochim. Acta 1998, 128, 37.

7. Fatibello-Filho, O.; Lupetti, K.O.; Vieira, I.C.; Talanta 2001, 55, 685.

8. Adams, R. N.; Anal. Chem. 1958, 30, 1576.

9. Matuszewski, W.; Trajanowicz, M.; Analyst 1988, 113, 735.
10. Ounigpipat W.; Alexander, P. W.; Southwell-Keely.; Anal. Chim. Acta 1995, $309,35$.

11. Wang, J.; Lin, M. S.; Electroanalysis 1989, 1, 43.

12. Dominguez-Sanches, P.; O’Sullivan, C.K.; Miranda-Ordieres, A. J.; Tu'nonBlanco, P.; Smyth, M. R.; Anal. Chim. Acta 1994, 291, 349.

13. Cheek, G.; Nelson, R. F.; Anal. Lett. 1978, 11, 393.

14. Gorton, L.; Electroanalysis 1995, 7, 23.

15. Petit, C.; Kauffmann, J. M.; Anal. Proc. 1995, 32, 11

16. Kalcher, K.; Kauffmann, J. M.; Wang, J.; Savancara, I.; Vytras, K.; Neuhold, C.; Yang, Z.; Electroanalysis 1995, 7, 5.

17. Ruzgas, T.; Csöregi, E.; Emnéus, J.; Gorton, L.; Marko-Varga, G.; Anal. Chim. Acta 1996, 330, 123.

18. Robinson, D. S. Em Oxidative Enzymes in Food; Robinson, D. S.; Eskin, N. A. M., eds.; Elsevier Applied Science: New York, 1991.

19. Bruemmer, J. H.; Roe, B.; Bowen, E. R.; J. Food Sci. 1976, 41,186.

20. Floris, G.; Medda, R.; Rinaldi, A.; Phytochemistry 1984, 23, 1527.

21. Lima, A. W. O.; Nascimento, V. B.; Pedrotti, J. J.; Angnes, L.; Anal. Chim. Acta 1997, 354, 325.

22. Signori, C. A.; Fatibello-Filho, O.; Quim. Nova 1994, 17, 38.

23. Vieira, I. C.; Fatibello-Filho, O.; Anal. Lett. 1997, 30, 895.

24. Fatibello-Filho, O.; Vieira, I. C.; Analyst 1997, 122, 345.

25. Fatibello-Filho, O.; Vieira, I. C.; Anal. Chim. Acta 1997, 354, 51.

26. Vieira, I. C.; Fatibello-Filho, O.; Anal. Chim. Acta 1998, 366, 111.

27. Vieira, I. C.; Fatibello-Filho, O.; Talanta 1998, 46, 559.

28. Vieira, I. C.; Fatibello-Filho, O.; Analyst 1998, 123, 1809.

29. Caruso, C. S.; Vieira, I. C.; Fatibello-Filho, O.; Anal. Lett. 1999, 32, 39

30. Gornall, A. G.; Bardawill, C. J.; David, M. M.; J. Biol. Chem. 1949, 177, 751.

31. Andersen, R. A.; Sowers, J. A.; Phytochemistry 1968, 7, 293.

32. Loomis, W. D.; Methods in Enzymology 1969, 13, 555.

33. Loomis, W. D.; Battaile, J.; Phytochemistry 1966, 5, 423.

34. Lourenço, E. J.; Neves, V. A.; Silva, M. A. J.; J. Agric. Food Chem. 1992, 40, 2369.

35. Sanderson, G. W.; Biochim. Biophys. Acta 1964, 92, 622.

36. Park, E. Y.; Luh, B. S.; J. Food Sci. 1985, 50, 678. 